\title{
Inclusive three jet production at the LHC at 7 and 13 TeV collision energies
}

\section{G. Chachamis*}

Instituto de Física Teórica UAM/CSIC, Nicolás Cabrera 15

\& Universidad Autónoma de Madrid, E-28049 Madrid, Spain. E-mail:

chachamisegmail.com

\section{F. Caporale}

Instituto de Física Teórica UAM/CSIC, Nicolás Cabrera 15

\& Universidad Autónoma de Madrid, E-28049 Madrid, Spain. E-mail:

francesco.caporale@uam.es

\section{F. G. Celiberto}

Dipartimento di Fisica, Università della Calabria \&

Istituto Nazionale di Fisica Nucleare, Gruppo Collegato di Cosenza,

I-87036 Arcavacata di Rende, Cosenza, Italy. E-mail:

francescogiovanni.celiberto@fis.unical.it

\section{Gordo Gómez}

Instituto de Física Teórica UAM/CSIC, Nicolás Cabrera 15

\& Universidad Autónoma de Madrid, E-28049 Madrid, Spain. E-mail:

david.gordo@csic.es

\section{A. Sabio Vera}

Instituto de Física Teórica UAM/CSIC, Nicolás Cabrera 15

\& Universidad Autónoma de Madrid, E-28049 Madrid, Spain. E-mail:

a.sabio.vera@gmail.com

We discuss briefly a recent study of new observables in LHC inclusive events with three tagged jets. One jet is in the forward direction, the second is in the backward direction and well-separated in rapidity from the first, whereas, the third tagged jet is to be found in more central regions of the detector. Taking into consideration that non-tagged mini-jet emissions are allowed and that they may be accounted for by the BFKL gluon Green function, we project the cross sections on azimuthal-angle components and define suitable ratios based on these projections which can provide several distinct tests of the BFKL dynamics.

XXIV International Workshop on Deep-Inelastic Scattering and Related Subjects

11-15 April, 2016

DESY Hamburg, Germany

\footnotetext{
* Speaker.
} 


\section{Introduction}

At hadronic colliders, when jets are produced at large relative rapidities the Balitsky-FadinKuraev-Lipatov (BFKL) framework in the leading logarithmic (LL) $[1,2,3,4,5,6]$ and next-toleading logarithmic (NLL) approximation [7, 8] is applicable. Mueller-Navelet jets [9], in particular ratios of projections on azimuthal-angle observables $\mathscr{R}_{n}^{m}=\langle\cos (m \phi)\rangle /\langle\cos (n \phi)\rangle$, for the azimuthal-angle formed by the two tagged jets, $\phi$, is an important example in which comparison of different NLL predictions with LHC experimental data has been quite successful $[10,11,12,13$, $14,15,16,17,18,19,20,21,22,23,24,25,26,27,28,29]$.

Recently, we proposed new observables for processes at the LHC that may be considered as a generalisation of the Mueller-Navelet jets. These processes are inclusive three-jet $[30,31]$ and four-jet production $[32,33]$. Here we will solely focus on the three-jet observables. These are based on the recently defined ratios [30]

$$
\mathscr{R}_{P Q}^{M N}=\frac{\left\langle\cos \left(M \phi_{1}\right) \cos \left(N \phi_{2}\right)\right\rangle}{\left\langle\cos \left(P \phi_{1}\right) \cos \left(Q \phi_{2}\right)\right\rangle},
$$

where $\phi_{1}$ and $\phi_{2}$ are, respectively, the azimuthal angle difference between the first and the second (central) jet and between this one and the third jet. The "observables" $\mathscr{R}_{P Q}^{M N}$ in Eq. (1.1) are defined at partonic level and cannot be readily compared to experimental data. For that reason, we report here on the hadronic level observables $R_{P Q}^{M N}$ [31]. This will allow for a comparison of our predictions with forthcoming analyses of the LHC experimental data. Within the collinear factorization scheme we produce the two utmost in rapidity jets and we associate to each one of them a forward "jet vertex" [34] before we connect these vertices with the central jet via two BFKL gluon Green functions. Finally we convolute the partonic differential cross-section with collinear parton distribution functions and we integrate over the momenta of all produced jets, using LHC experimental cuts, to compute the ratios $R_{P Q}^{M N}$. We fix the rapidity of the central jet to lie in the middle of the two utmost tagged jets.

\section{Hadronic inclusive three-jet production in multi-Regge kinematics}

Let us recapitulate some of the notation used in [30, 31]. If the transverse momenta of the utmost jets are $\vec{k}_{A, B}$ and their rapidity distance $Y$ is large while the central jet has transverse momentum $\vec{k}_{J}$ and mini-jet activity is allowed between the three tagged jets, the studied process is

$$
\operatorname{proton}\left(p_{1}\right)+\operatorname{proton}\left(p_{2}\right) \rightarrow \operatorname{jet}\left(k_{A}\right)+\operatorname{jet}\left(k_{J}\right)+\operatorname{jet}\left(k_{B}\right)+\text { minijets . }
$$

The projection on azimuthal-angle components can give the mean value (with $M, N$ being positive integers)

$$
\begin{aligned}
& \mathscr{C}_{M N}=\left\langle\cos \left(M\left(\theta_{A}-\theta_{J}-\pi\right)\right) \cos \left(N\left(\theta_{J}-\theta_{B}-\pi\right)\right)\right\rangle \\
= & \frac{\int_{0}^{2 \pi} d \theta_{A} d \theta_{B} d \theta_{J} \cos \left(M\left(\theta_{A}-\theta_{J}-\pi\right)\right) \cos \left(N\left(\theta_{J}-\theta_{B}-\pi\right)\right) d \sigma^{3-\text { jet }}}{\int_{0}^{2 \pi} d \theta_{A} d \theta_{B} d \theta_{J} d \sigma^{3-\text { jet }}}
\end{aligned}
$$

where we define the two relative azimuthal angles between each external jet and the central one as $\Delta \theta_{\widehat{A J}}=\theta_{A}-\theta_{J}-\pi$ and $\Delta \theta_{\widehat{J B}}=\theta_{J}-\theta_{B}-\pi$ respectively and $d \sigma^{3-\text { jet }}$ is defined in [30]. 
As we mentioned previously, our main target is to provide theoretical estimates that can be compared against current and future experimental data, therefore, we introduce kinematical cuts already in use at the LHC. We integrate $\mathscr{C}_{M, N}$ over the momenta of the tagged jets in the form

$$
C_{M N}=\int_{Y_{A}^{\min }}^{Y_{A}^{\max }} d Y_{A} \int_{Y_{B}^{\min }}^{Y_{B}^{\max }} d Y_{B} \int_{k_{A}^{\min }}^{k_{A}^{\max }} d k_{A} \int_{k_{B}^{\min }}^{k_{B}^{\max }} d k_{B} \int_{k_{J}^{\min }}^{k_{J}^{\max }} d k_{J} \delta\left(Y_{A}-Y_{B}-Y\right) \mathscr{C}_{M N}
$$

where the rapidities of the utmost jet rapidities take values in the range $Y_{A, B}^{\min }=-4.7$ and $Y_{A, B}^{\max }=4.7$ while their difference $Y \equiv Y_{A}-Y_{B}$ is kept fixed at definite values in the range $5<Y<9$. We compute for two different center-of-mass energies, $\sqrt{s}=7$ and $\sqrt{s}=13 \mathrm{TeV}$, and we use both a symmetric and an asymmetric cut $[19,27]$ :

1. $k_{A}^{\min }=35 \mathrm{GeV}, k_{B}^{\min }=35 \mathrm{GeV}, k_{A}^{\max }=k_{B}^{\max }=60 \mathrm{GeV}$ (symmetric);

2. $k_{A}^{\min }=35 \mathrm{GeV}, k_{B}^{\min }=50 \mathrm{GeV}, k_{A}^{\max }=k_{B}^{\max }=60 \mathrm{GeV}$ (asymmetric).

Seeking the best possible perturbative stability in our results (see [15] for a related discussion). we remove the zeroth conformal spin contribution of the BFKL kernel. by introducing the ratios

$$
R_{P Q}^{M N}=\frac{C_{M N}}{C_{P Q}}, \quad M, N, P, Q>0,
$$

which are free from any $n=0$ dependence. Thus, we can study the $\operatorname{ratios} R_{P Q}^{M N}(Y)$ in Eq. (2.4) as functions of the rapidity difference $Y$ between the utmost jets for a set of typical values of $M, N, P, Q$. The momentum of the central jet is permitted to take values in three different domains: $\left[20 \mathrm{GeV}<k_{J}<35 \mathrm{GeV}\right]$ (smaller that $k_{A}, k_{B}$ ), [35 GeV $\left.<k_{J}<60 \mathrm{GeV}\right]$ (similar to $k_{A}, k_{B}$ ) and $\left[60 \mathrm{GeV}<k_{J}<120 \mathrm{GeV}\right]$ (larger than $k_{A}, k_{B}$ ). This allows us to see how the ratio $R_{P Q}^{M N}(Y)$ changes behaviour depending on the relative size of the three jets, see Fig. 1 for the behaviour of $\mathscr{R}_{22}^{11}$.

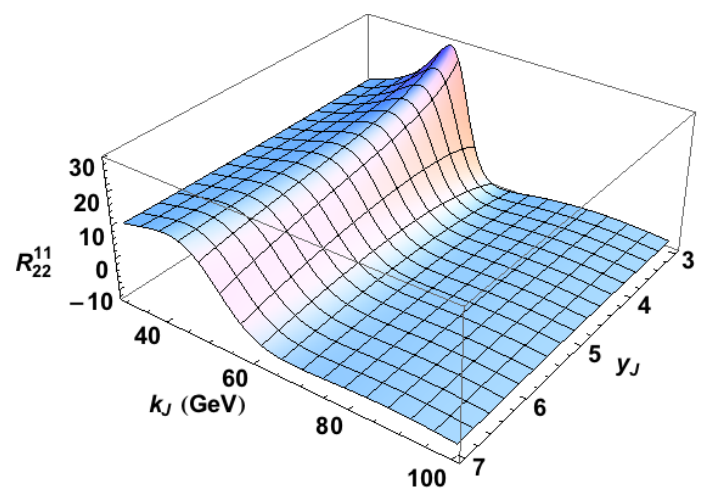

Figure 1: $3 \mathrm{D}$ plot of the partonic $\mathscr{R}_{22}^{11}$ as a function of the momentum $k_{J}$ and the rapidity $y_{J}$ of the central jet for $k_{A}=40 \mathrm{GeV}, k_{B}=50 \mathrm{GeV}$ and $\Delta Y_{A, B}=10$.

In total, we have computed the results for six different ratios in [31], here we are only showing for $R_{22}^{11}$ in Fig. 2. Generally, the dependence of the different observables on the rapidity difference between $k_{A}$ and $k_{B}$ is rather smooth. The slope of the three curves, in absolute values, depends on the particular observable. Another interesting observation is that there are ratios for which changing from the symmetric to the asymmetric cut makes no real difference and other ratios for which the 

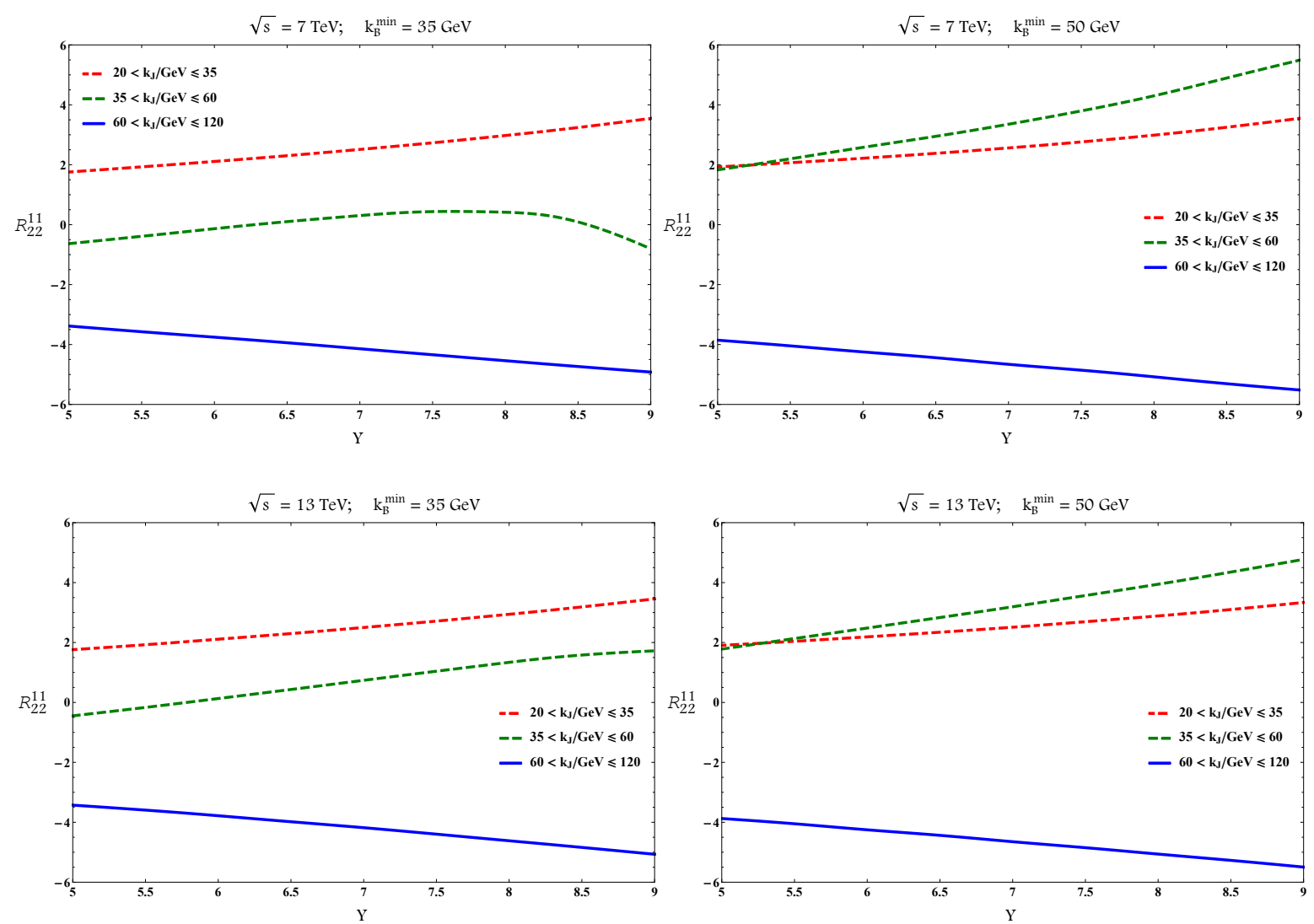

Figure 2: $Y$-dependence of $R_{22}^{11}$ for $\sqrt{s}=7 \mathrm{TeV}$ (top) and $\sqrt{s}=13 \mathrm{TeV}$ (bottom). $k_{B}^{\text {min }}=35 \mathrm{GeV}$ (left column) and $k_{B}^{\mathrm{min}}=50 \mathrm{GeV}$ (right column).

picture changes drastically. The most important thing to note though would be that, in general, for most of the ratios there are no large scale changes when we go up to $\sqrt{s}=13 \mathrm{TeV}$ from $\sqrt{s}=7 \mathrm{TeV}$. This is indeed significant as it seemingly suggests that we reach a sort of asymptotic regime for the kinematical configurations used in our study. It also indicates that our ratios are indeed mostly insensitive to effects stemming from outside the BFKL dynamics and which normally cannot be detached (e.g. influence from the PDFs).

\section{Summary \& Outlook}

We have discussed a first phenomenological study at hadronic level of azimuthal-angle dependent observables in inclusive three-jet production at the LHC within the BFKL resummation program. The study was focused on how the ratios $R_{M N}^{P Q}$, behave when we change the rapidity difference $Y$ between the utmost jets from 5 to 9 units. In general, a smooth functional dependence of the ratios on $Y$ is seen. A major observation is that these observables do not have a significantly different behaviour after raising the colliding energy from 7 to $13 \mathrm{TeV}$ which make us confident that they highlight the most important features of the tagged jets azimuthal behavior within the BFKL dynamics. It will be important to see whether fixed order calculations and studies with the BFKL inspired Monte Carlo BFKLex [35, 36, 37, 38, 39, 40, 41]. give similar predictions. Estimates from the usual all-purpose collinear Monte Carlo tools are also needed to have a full picture from 
the theoretical side. In conclusion, only an experimental analysis for these observables using existing and future LHC data will show us whether we can use these observables as a new probe for the BFKL dynamics and if so, it will help toward the direction of assessing the window of applicability of the BFKL resummation program.

\section{Acknowledgements}

GC acknowledges support from the MICINN, Spain, under contract FPA2013-44773-P. ASV acknowledges support from Spanish Government (MICINN (FPA2010-17747,FPA2012-32828)) and, together with FC and FGC, to the Spanish MINECO Centro de Excelencia Severo Ochoa Programme (SEV-2012-0249). FGC thanks the Instituto de Física Teórica (IFT UAM-CSIC) in Madrid for warm hospitality.

\section{References}

[1] L. N. Lipatov, Sov. Phys. JETP 63 (1986) 904 [Zh. Eksp. Teor. Fiz. 90 (1986) 1536].

[2] I. I. Balitsky and L. N. Lipatov, Sov. J. Nucl. Phys. 28 (1978) 822 [Yad. Fiz. 28 (1978) 1597].

[3] E. A. Kuraev, L. N. Lipatov and V. S. Fadin, Sov. Phys. JETP 45 (1977) 199 [Zh. Eksp. Teor. Fiz. 72 (1977) 377].

[4] E. A. Kuraev, L. N. Lipatov and V. S. Fadin, Sov. Phys. JETP 44 (1976) 443 [Zh. Eksp. Teor. Fiz. 71 (1976) 840] [Erratum-ibid. 45 (1977) 199].

[5] L. N. Lipatov, Sov. J. Nucl. Phys. 23 (1976) 338 [Yad. Fiz. 23 (1976) 642].

[6] V. S. Fadin, E. A. Kuraev and L. N. Lipatov, Phys. Lett. B 60 (1975) 50.

[7] V. S. Fadin and L. N. Lipatov, Phys. Lett. B 429 (1998) 127 [hep-ph/9802290].

[8] M. Ciafaloni and G. Camici, Phys. Lett. B 430 (1998) 349 [hep-ph/9803389].

[9] A. H. Mueller and H. Navelet, Nucl. Phys. B 282 (1987) 727.

[10] V. Del Duca and C. R. Schmidt, Phys. Rev. D 49 (1994) 4510 [hep-ph/9311290].

[11] W. J. Stirling, Nucl. Phys. B 423 (1994) 56 [hep-ph/9401266].

[12] L. H. Orr and W. J. Stirling, Phys. Rev. D 56 (1997) 5875 [hep-ph/9706529].

[13] J. Kwiecinski, A. D. Martin, L. Motyka and J. Outhwaite, Phys. Lett. B 514 (2001) 355 [hep-ph/0105039].

[14] M. Angioni, G. Chachamis, J. D. Madrigal and A. Sabio Vera, Phys. Rev. Lett. 107, 191601 (2011) doi:10.1103/PhysRevLett.107.191601 [arXiv:1106.6172 [hep-th]].

[15] F. Caporale, B. Murdaca, A. Sabio Vera and C. Salas, Nucl. Phys. B 875 (2013) 134 [arXiv:1305.4620 [hep-ph]].

[16] F. Caporale, D. Y. Ivanov, B. Murdaca and A. Papa, Nucl. Phys. B 877 (2013) 73 [arXiv:1211.7225 [hep-ph]].

[17] C. Marquet and C. Royon, Phys. Rev. D 79, 034028 (2009) doi:10.1103/PhysRevD.79.034028 [arXiv:0704.3409 [hep-ph]].

[18] D. Colferai, F. Schwennsen, L. Szymanowski and S. Wallon, JHEP 1012, 026 (2010) doi:10.1007/JHEP12(2010)026 [arXiv:1002.1365 [hep-ph]]. 
[19] B. Ducloue, L. Szymanowski and S. Wallon, JHEP 1305, 096 (2013) doi:10.1007/JHEP05(2013)096 [arXiv:1302.7012 [hep-ph]].

[20] B. Ducloue, L. Szymanowski and S. Wallon, Phys. Lett. B 738, 311 (2014) doi:10.1016/j.physletb.2014.09.025 [arXiv:1407.6593 [hep-ph]].

[21] A. H. Mueller, L. Szymanowski, S. Wallon, B. W. Xiao and F. Yuan, JHEP 1603, 096 (2016) doi:10.1007/JHEP03(2016)096 [arXiv:1512.07127 [hep-ph]].

[22] A. Sabio Vera, Nucl. Phys. B 746 (2006) 1 [hep-ph/0602250].

[23] A. Sabio Vera and F. Schwennsen, Nucl. Phys. B 776 (2007) 170 [hep-ph/0702158 [HEP-PH]].

[24] B. Ducloue, L. Szymanowski and S. Wallon, Phys. Rev. Lett. 112 (2014) 082003 [arXiv:1309.3229 [hep-ph]].

[25] F. Caporale, D. Y. Ivanov, B. Murdaca and A. Papa, Eur. Phys. J. C 74 (2014) 3084 [arXiv:1407.8431 [hep-ph]].

[26] F. Caporale, D. Y. Ivanov, B. Murdaca and A. Papa, Phys. Rev. D91 (2015) 11, 114009 [arXiv:1504.06471 [hep-ph]].

[27] F. G. Celiberto, D. Yu. Ivanov, B. Murdaca and A. Papa, Eur. Phys. J. C 75 (2015) 292 [arXiv:1504.08233 [hep-ph]].

[28] F. G. Celiberto, D. Y. Ivanov, B. Murdaca and A. Papa, arXiv:1604.08013 [hep-ph].

[29] F. G. Celiberto, D. Y. Ivanov, B. Murdaca and A. Papa, Eur. Phys. J. C 76, no. 4, 224 (2016) doi:10.1140/epjc/s10052-016-4053-5 [arXiv:1601.07847 [hep-ph]].

[30] F. Caporale, G. Chachamis, B. Murdaca and A. Sabio Vera, Phys. Rev. Lett. 116, no. 1, 012001 (2016) doi:10.1103/PhysRevLett.116.012001 [arXiv:1508.07711 [hep-ph]].

[31] F. Caporale, F. G. Celiberto, G. Chachamis, D. G. Gomez and A. Sabio Vera, arXiv:1603.07785 [hep-ph].

[32] F. Caporale, F. G. Celiberto, G. Chachamis and A. Sabio Vera, Eur. Phys. J. C 76, no. 3, 165 (2016) doi:10.1140/epjc/s10052-016-3963-6 [arXiv:1512.03364 [hep-ph]].

[33] F. Caporale, F. G. Celiberto, G. Chachamis, D. G. Gomez and A. Sabio Vera, arXiv:1606.00574 [hep-ph].

[34] F. Caporale, D. Yu. Ivanov, B. Murdaca, A.Papa, A.Perri, JHEP 1202 (2012) 101; [arXiv:1212.0487 [hep-ph]].

[35] G. Chachamis, M. Deak, A. Sabio Vera and P. Stephens, Nucl. Phys. B 849 (2011) 28 [arXiv:1102.1890 [hep-ph]].

[36] G. Chachamis and A. Sabio Vera, Phys. Lett. B 709 (2012) 301 [arXiv:1112.4162 [hep-th]].

[37] G. Chachamis and A. Sabio Vera, Phys. Lett. B 717 (2012) 458 [arXiv:1206.3140 [hep-th]].

[38] G. Chachamis, A. Sabio Vera and C. Salas, Phys. Rev. D 87 (2013) 1, 016007 [arXiv:1211.6332 [hep-ph]].

[39] F. Caporale, G. Chachamis, J. D. Madrigal, B. Murdaca and A. Sabio Vera, Phys. Lett. B 724 (2013) 127 [arXiv:1305.1474 [hep-th]].

[40] G. Chachamis and A. Sabio Vera, arXiv:1511.03548 [hep-ph].

[41] G. Chachamis and A. Sabio Vera, JHEP 1602 (2016) 064 doi:10.1007/JHEP02(2016)064 [arXiv:1512.03603 [hep-ph]]. 